\title{
Wnt signaling suppresses MAPK-driven proliferation of intestinal stem cells
}

\author{
Zahra Kabiri, ${ }^{1}$ Gediminas Greicius, ${ }^{2}$ Hamed Zaribafzadeh, ${ }^{1}$ Amanda Hemmerich, ${ }^{3}$ Christopher M. Counter, ${ }^{1,4}$ and David M. Virshup ${ }^{2,5}$ \\ 'Department of Pharmacology and Cancer Biology, Duke University Medical Center, Durham, North Carolina, USA. Program in Cancer and Stem Cell Biology, Duke-NUS Medical School, Singapore. \\ ${ }^{3}$ Department of Pathology, Duke University Medical Center, Durham, North Carolina, USA. ${ }^{4}$ Department of Radiation Oncology, Duke University Medical Center, Durham, North Carolina, USA. ${ }^{5}$ Department of \\ Pediatrics, Duke University School of Medicine, Durham, North Carolina, USA.
}

\begin{abstract}
Intestinal homeostasis depends on a slowly proliferating stem cell compartment in crypt cells, followed by rapid proliferation of committed progenitor cells in the transit amplifying (TA) compartment. The balance between proliferation and differentiation in intestinal stem cells (ISCs) is regulated by Wnt/ $\beta$-catenin signaling, although the mechanism remains unclear. We previously targeted PORCN, an enzyme essential for all Wnt secretion, and demonstrated that stromal production of Wnts was required for intestinal homeostasis. Here, a PORCN inhibitor was used to acutely suppress Wnt signaling. Unexpectedly, the treatment induced an initial burst of proliferation in the stem cell compartment of the small intestine, due to conversion of ISCs into TA cells with a loss of intrinsic ISC self-renewal. This process involved MAPK pathway activation, as the proliferating cells in the base of the intestinal crypt contained phosphorylated ERK1/2, and a MEK inhibitor attenuated the proliferation of ISCs and their differentiation into TA cells. These findings suggest a role for Wnt signaling in suppressing the MAPK pathway at the crypt base to maintain a pool of ISCs. The interaction between Wnt and MAPK pathways in vivo has potential therapeutic applications in cancer and regenerative medicine.
\end{abstract}

\section{Introduction}

The intestine is the most proliferative tissue in the body, as the epithelial layer of the small intestine renews itself every 3-5 days. Intestinal stem cells (ISCs) in the base of intestinal crypts tightly control this process by producing intestinal progenitors known as transit amplifying (TA) cells, which are located above the stem cells in the crypt. These progenitor cells proliferate and differentiate into all absorptive and secretary cells. Multiple pathways including NOTCH, MAPK, WNT, HIPPO, and BMP signaling coordinate orderly proliferation and differentiation to maintain continuous production of the diverse cell types of the intestinal epithelium. Among these regulatory pathways, Wnt/ $\beta$-catenin signaling plays a pivotal role to maintain intestinal stem pools. Targeting Wnt signaling by diverse means including overexpression of DKK1 (1), inhibition of RSPOs (2, 3), knockout of TCF4 or $\beta$-catenin in the epithelial cells, or inhibition of PORCN in the stroma impairs the function of small ISCs and blocks proliferation following 5-7 days of treatment (4-7). It is therefore widely held that Wnt signaling facilitates both stemness and proliferation. However, establishing the direct role of Wnts is challenging in a system with multiple interacting components. In fact, other signaling pathways such as MAPK (8-10) and even PDG2 (11) also impact the proliferation of intestinal stem and progenitor cells. Wnt signaling might interact with these additional

Authorship note: ZK and GG contributed equally to this work. Conflict of interest: The authors have declared that no conflict of interest exists. Submitted: December 26, 2017; Accepted: June 12, 2018. Reference information: / Clin Invest. 2018;128(9):3806-3812. https://doi.org/10.1172/JCI99325 pathways in a direct or indirect manner. It is difficult to tease out dependencies and causality in single time point, long-term assays. Indeed, little is known about the immediate effect of Wnt inhibition on ISC proliferation or the TA cells, even though this is likely to be the critical time point for the subsequent collapse of the ISC compartment.

Here, we used a PORCN inhibitor $(6,12)$ to acutely inhibit secretion of all Wnts and test the role of Wnts in ISC proliferation. Our results show that Wnt signaling suppresses both proliferation and MAPK signaling in ISCs. This serves to maintain stem cell pools by preventing their differentiation into TA cells. These results clarify the role of Wnt signaling in the intestine. Combination therapy with Wnt and MEK inhibitors might provide added efficacy in select Wnt-addicted cancers.

\section{Results and Discussion}

To examine the early effects of withdrawal of Wnt signaling in ISC proliferation, we selected a dose of the PORCN inhibitor C59 that impaired intestinal homeostasis within 10-15 days (6). C57/ BL6 mice were administered a single dose of C59 $(100 \mathrm{mg} / \mathrm{kg})$ or vehicle as control by oral gavage and sacrificed 24 hours later. Proliferating intestinal cells were assessed using EdU incorporation. As expected, EdU incorporation was seen primarily in TA cells of vehicle-treated mice (Figure 1A and Supplemental Figure 1C; supplemental material available online with this article; https:// doi.org/10.1172/JCI99325DS1). Unexpectedly, we observed a significant increase in proliferating cells in crypt base cells in the duodenum, jejunum, and ileum in C59-treated mice (Figure 1A, Supplemental Figure 1A, and quantified in Figure 1B). To independently validate this finding, intestinal samples from these mice 
A
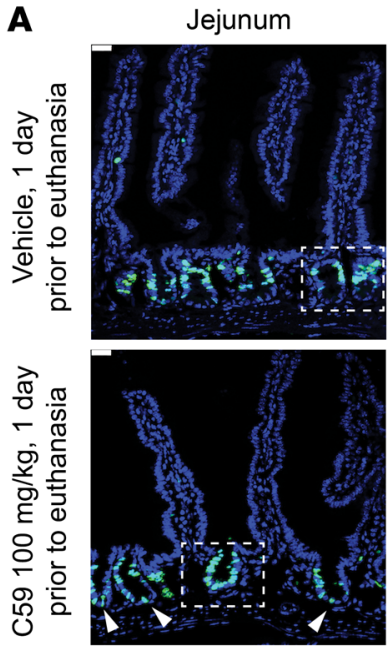

B

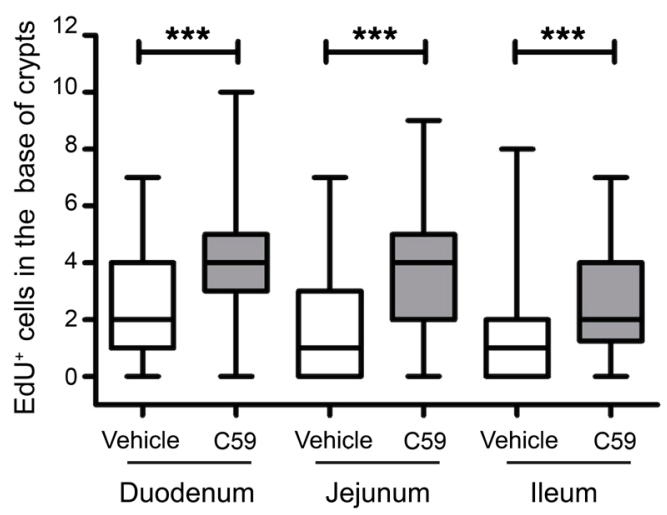

DAPI/EdU
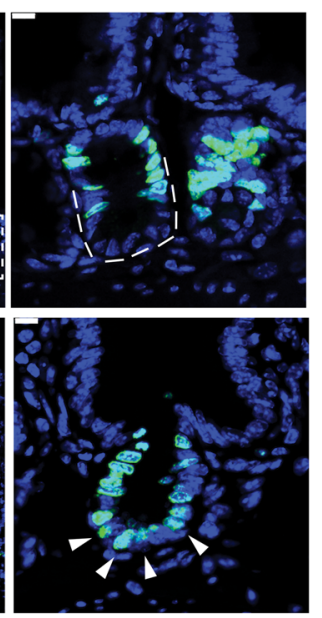

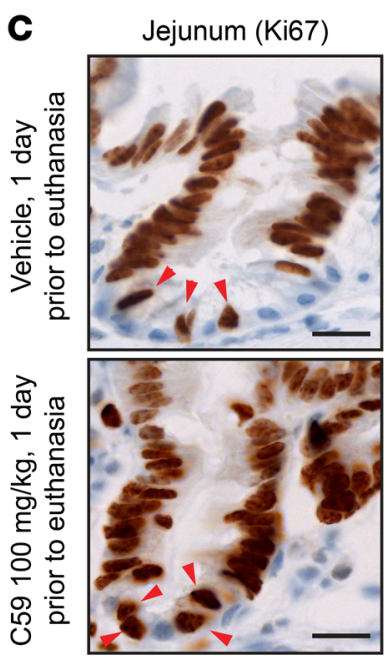

E

E Jejunum (CAS3)

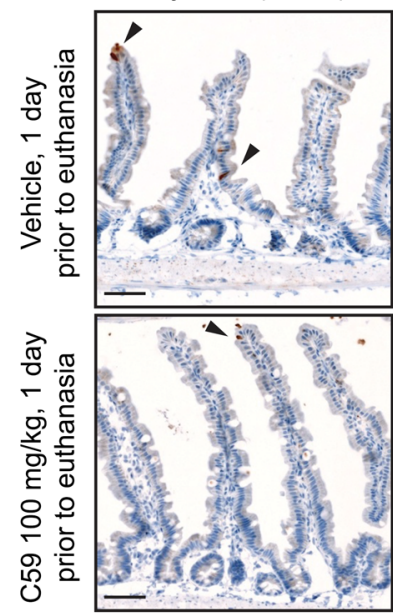

D

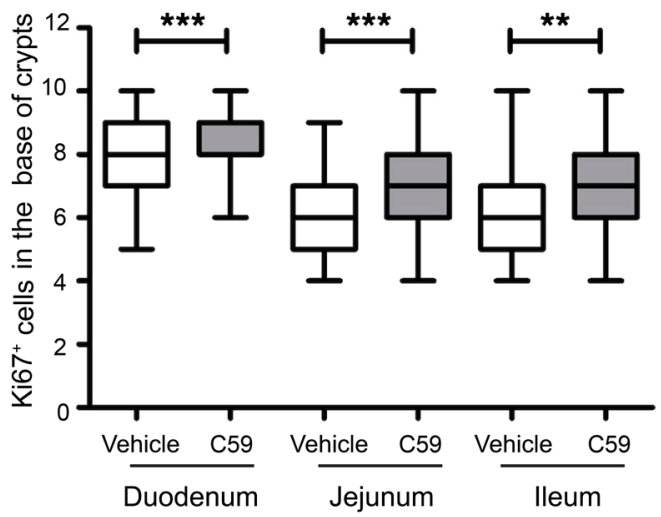

Figure 1. Wht inhibition enhances proliferation of intestinal stem cells. (A) C59 induced proliferation in the crypt base. Representative images of jejunal samples from mice treated with 1 dose of vehicle or $C 59(100 \mathrm{mg} / \mathrm{kg}) 1$ day prior to euthanasia. Proliferative cells were marked with EdU given 2 hours prior to euthanasia and DAPI stained the nuclei of cells. Arrows indicate EdU+ cells in crypt base. Scale bar, $25 \mu \mathrm{m}$. Image on right is higher magnification of outlined area of crypt (scale bar, $10 \mu \mathrm{m}$ ). (B) Quantification of EdU+ cells within 10 counted cells in the crypt base. Fifteen to twenty crypts were counted for each region of each mouse intestine (vehicle, $n=9$ mice; C59, $n=8$ mice; 3 experimental replicates). (C) Representative images of Ki67 staining in the vehicle- or [59-treated mice. Scale bar, $20 \mu \mathrm{m}$. Arrows indicate Ki67 ${ }^{+}$cells in the crypt base. (D) Enrichment of Ki67 cells in the crypt base of vehicle- versus C59-treated mice. Twenty crypts were counted for each region of intestine per mouse (vehicle, $n=4 ;$ C59, $n=7 ; 2$ experimental replicates). (E) C59 does not induce apoptosis in intestinal crypts. Representative images of cleaved-caspase 3 (CAS3) staining in jejunal sections of mice treated as described above. Arrows mark the apoptotic cells in villi as an internal positive control. Scale bar, $50 \mu \mathrm{m} .{ }^{* *} P<0.001$, Mann-Whitney $U$ test.

were stained for Ki67. Similar to what was seen with EdU, the fraction of crypt base cells positive for Ki67 increased upon C59 treatment. (Figure 1, C and D, and Supplemental Figure 1D).

The observed proliferation in the stem cell compartment at the base of the crypt in response to C59 could be generated by different biological mechanisms. One trivial explanation is that PORCN inhibition is proapoptotic for ISCs and thus TA cells simply moved down toward the base of the crypt. To test this possibility, intestinal samples were stained with antibodies against cleaved-caspase 3 (CAS3). As shown in Figure 1E and Supplemental Figure 1E, no apoptotic cells $\left(\mathrm{CAS3}^{+}\right)$were detected in the crypt base of either vehicle- or C59-treated samples. This suggests that Wnt inhibition instead promotes ISC proliferation.

This proliferation phenotype could be a product of ISC differentiation. Thus, we performed lineage tracing to determine the fate of ISC cells after Wnt inhibition. Wnt-dependent Lgr5 expression marks ISCs, which normally divide symmetrically to replenish the ISC pool and to generate new TA cells $(13,14)$. We therefore tested whether $\operatorname{Lgr} 5^{+}$ISCs survive after PORCN inhibition to become TA cells. Short-term lineage tracing was performed using Lgr5-EGFP-IRES-CreERT2/Rosa-LSL-tdTomato mice to follow the fate of intestinal Lgr5-expressing cells. Mice were treated with tamoxifen to label $\operatorname{Lgr} 5^{+}$cells with tdTomato, followed by C59 treatment, after which the intestines were removed for analysis.

In pilot studies, labeling was evaluated 24 hours after tamoxifen administration. Occasional crypts contained single cells that were positive for Rosa-tdTomato, but not for Lgr5-EGFP, indicating that TA cells can emerge from $\operatorname{Lgr} 5^{+}$cells within this time frame (Supplemental Figure 3A). To avoid potential lineage tracing from newly generated TA cells, we administered the first dose of C59 12 hours after the tamoxifen and then continued daily C59 (100 mg/kg) treatment for 3 days (Figure 2A). These lineage-tracing experiments did not show any difference between C59- and 
$\mathbf{A}$

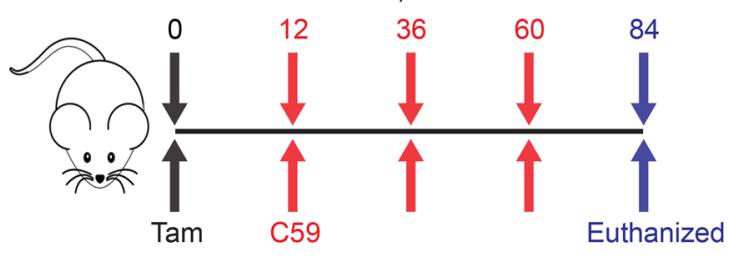

Dosage: $100 \mathrm{mg} / \mathrm{kg}$

B
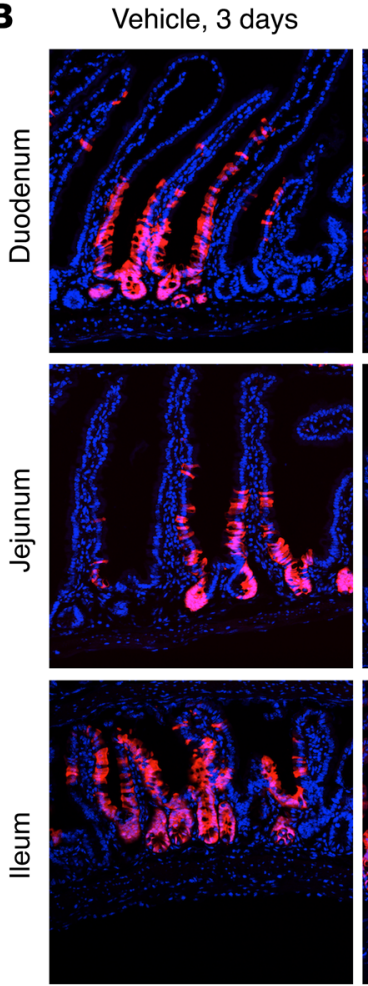

DAPI / Rosa-tomato (Red)

Lgr5-CreER ${ }^{\text {T2} / R o s a-L S L-t d T o m a t o ~}$
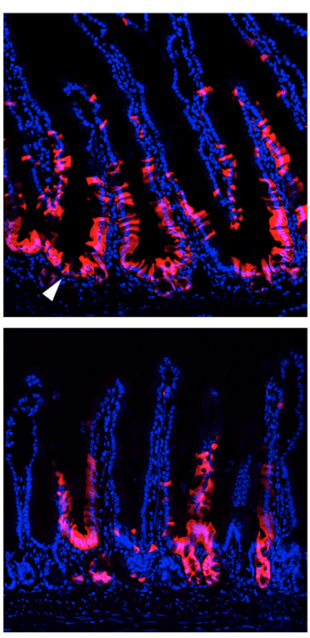

C

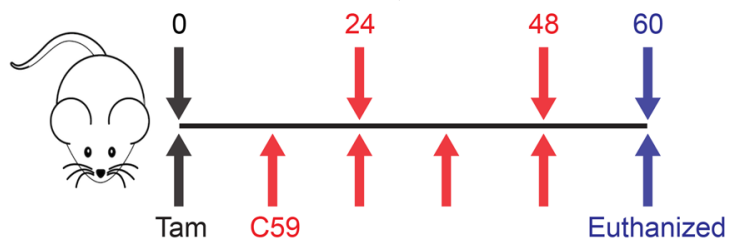

Dosage: $50 \mathrm{mg} / \mathrm{kg}$

D

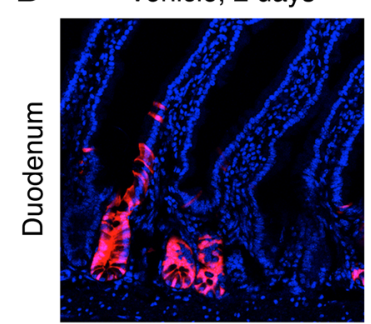

C59 BID, 2 days
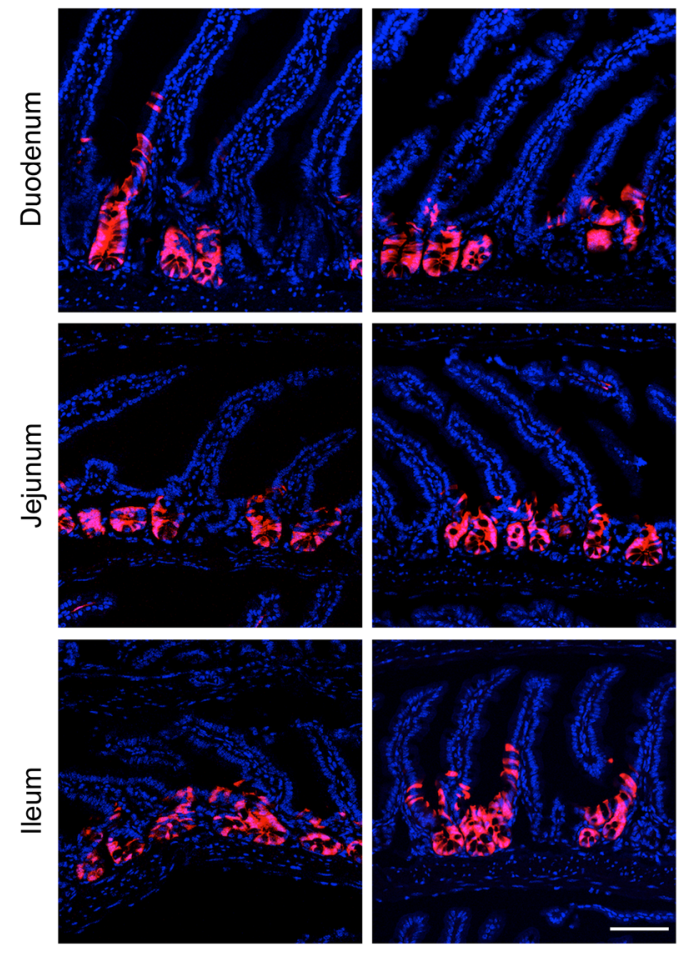

DAPI / Rosa-tomato (Red)

Lgr5-CreER ${ }^{\text {T2} / R o s a-L S L-t d T o m a t o ~}$

Figure 2. Passive lineage commitment of Lgr5 stem cells is intact after Wnt inhibition. (A and C) Drug dosing protocol. Lgr5-ECFP-IRES-CreER $R^{T 2} / R o s a-$ LSL-tdTomato mice were treated with tamoxifen and C59 according to the time line. (B) Wnt inhibition (C59 treatment with $100 \mathrm{mg} / \mathrm{kg}$, once daily [QD]) for 3 days does not block $L g r 5^{+}$lineage tracing. Representative images of positive lineage-traced cells from $L g r 5$ cells, which are marked by endogenous RosatdTomato (red), are shown for both vehicle- and C59-treated mice. (D) More intensive Wnt inhibition for 2 days still does not block $\mathrm{Lgr5^{+ }}$ lineage tracing. Representative images of lineage tracing in vehicle- and C59-treated mice $(50 \mathrm{mg} / \mathrm{kg}$; BID, twice daily) are shown. Scale bar, $100 \mu \mathrm{m}$.

vehicle-treated mice, suggesting that differentiation of ISCs into TA cells was unchanged in the absence of Wnt signaling (Figure 2, $\mathrm{A}$ and B, and Supplemental Figure $3 \mathrm{C}$ ).

The dosing schedule of C59 can have a time-dependent impact on Wnt activity in vivo. The in vivo half-life of C59 is approximately 2 hours (12), and consistent with previous reports with other PORCN inhibitors $(15,16)$, there was a rebound of Wnt target gene expression 1 day after the first dose of C59 (see Supplemental Figure 4A). If the proliferative burst in $L g r 5^{+}$cells is due to drug washout, the proliferative effect might be blunted by more frequent dosing. Conversely, if the suppression of Wnt signaling causes the increase in proliferation of $\operatorname{Lr} 5^{+}$cells, more frequent dosing would enhance the proliferation rate. Therefore, the experiment was repeated with mice dosed twice daily for a total daily dose of $100 \mathrm{mg} / \mathrm{kg}$ (50 mg/kg twice daily) as this high dose was previously shown to impair intestinal homeostasis within 5-7 days. A substantial increase in the number of proliferative cells was seen on the first 2 days of C59 treatment, and this was followed by the disappearance of proliferative cells by the fourth day (Supplemental Figure 2, A-C). Interestingly, we observed normal lineage tracing in the crypts of the C59-treated mice (Figure 2, C and D, and Supplemental Figure 3C). These findings support the conclusion that acute Wnt inhibition leads to enhanced ISC proliferation and unimpaired differentiation.

$\mathrm{Lgr}^{+}$cells expressing Olfm 4 and Ascl2 are an active type of ISC that can regenerate intestinal epithelial cells every 3-5 days $(14,17,18)$. In contrast, Bmil-expressing ISCs at the +4 position of the crypt are more quiescent and may serve as a reservoir to 
A
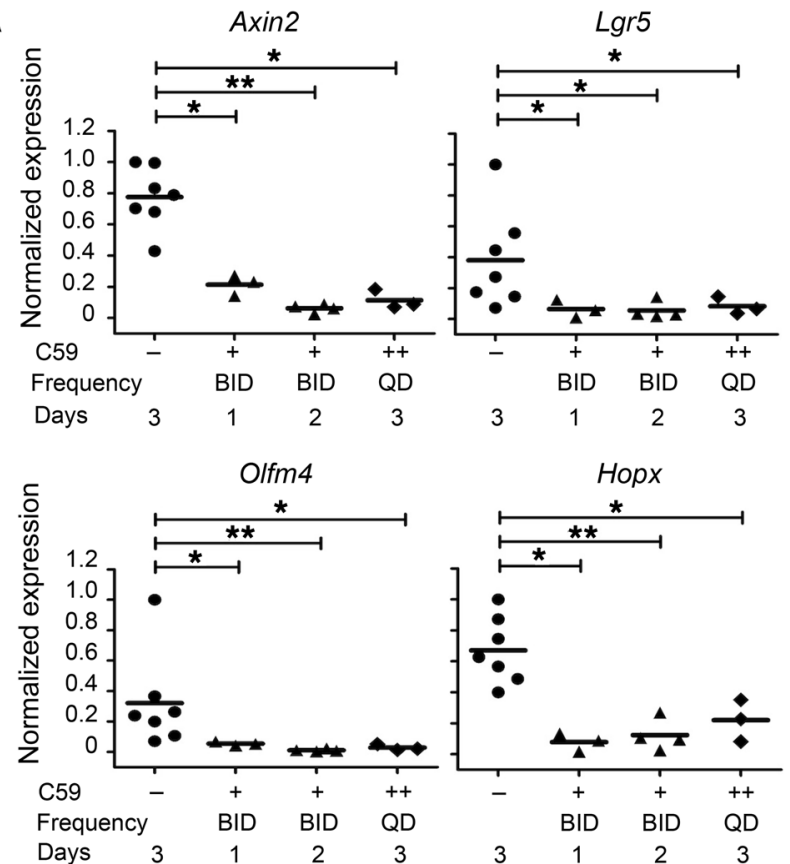
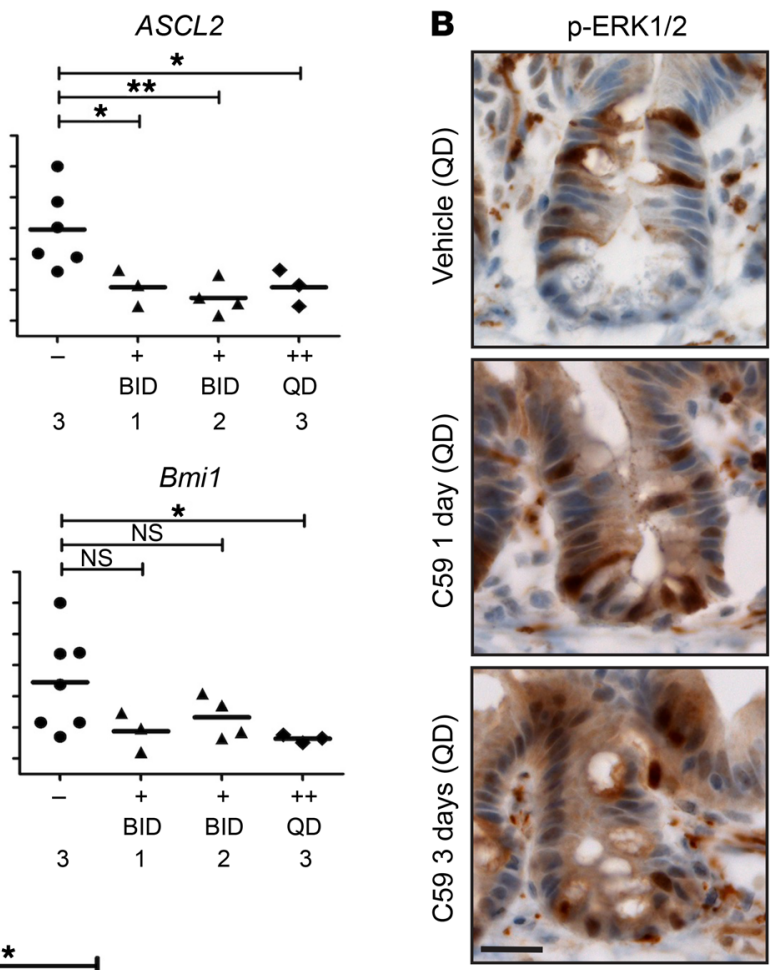

C

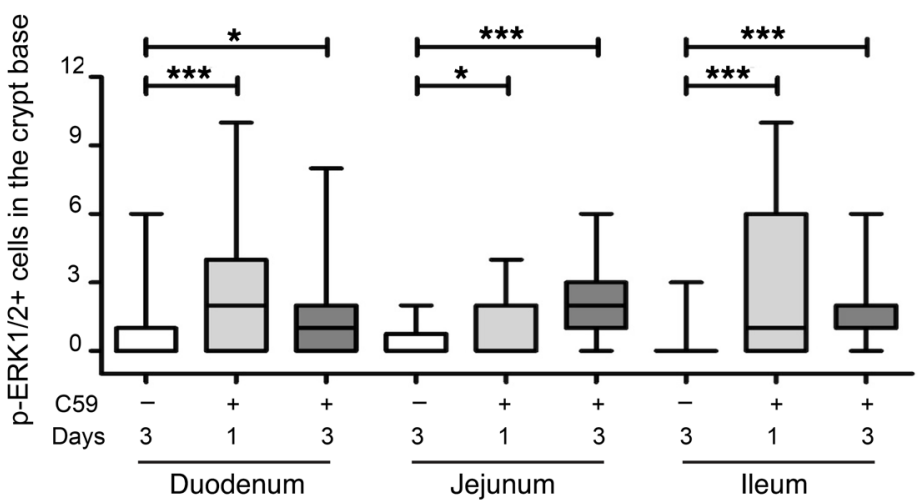

Figure 3. Wnt inhibition upregulates MAPK signaling in the crypt base. (A) PORCN inhibition suppresses expression of Wnt target genes and stem cell markers. Mice were administered C59 at the indicated dose and frequency (+, $50 \mathrm{mg} / \mathrm{kg}$ twice daily [BID]; ++, $100 \mathrm{mg} / \mathrm{kg}$ once daily [QD]), and quantitative RT-PCR was performed on RNA from duodenal samples. Expression of Axin2, Lgr5, Ascl2, Olfm4, Hopx, and Bmi1 was normalized to Pgk and Actb (actin). Each dot represents 1 mouse. (B) C59 treatment leads to increased ERK1/2 phosphorylation at the crypt base. Representative images of p-ERK1/2 staining in jejunum samples. Scale bar, $50 \mu \mathrm{m}$. (C) Quantification of $\mathrm{p}$-ERK1/2+ cells (cytoplasmic or nuclear staining) in crypt base of vehicle- or C59-treated mice (100 mg/kg, QD). NS, nonsignificant; ${ }^{*} P<0.05 ;{ }^{* *} P<0.01$; ${ }^{* *} P<0.001$, Mann-Whitney $U$ test.

regenerate the intestinal epithelial cells after severe damage (Supplemental Figure 3B) $(19,20)$. To test if Bmil ${ }^{+}$ISCs could account for the crypt cell proliferation after acute Wnt inhibition, Bmi1CreER ${ }^{T 2} /$ Rosa-LSL-tdTomato mice were treated with tamoxifen and then either $\mathrm{C} 59$ or vehicle according to the time line indicated in Supplemental Figure 3D. Under unstressed conditions, more tdTomato ${ }^{+}$cells appeared in the crypt base 3 days after tamoxifen injection. However, the C59-treated mice had significantly fewer labeled cells in the crypt base of the jejunum and ileum (Supplemental Figure 3, E and F). Thus, the proliferation in the crypt base after acute Wnt inhibition does not appear to be due to active regeneration by $\mathrm{Bmil}^{+}$ISC cells.

To examine the effect of PORCN inhibition on Wnt signaling, we determined the level of mRNA expression for Wnt target genes and stem cell markers by quantitative RT-PCR in vehicle- and C59-treat- ed mice. There was a significant reduction in the expression of Axin 2 (Wnt target) and the stem cell markers Lgr5, Olfm4, Ascl2, Hopx , and Bmil in the C59-treated mice as early as 1 day after the first dose (Figure 3A). Conversely, expression of intestinal differentiation markers was not affected during the course of this experiment (Supplemental Figure 4C). In addition, EdU staining in C59-treated Lgr5-EGFPCreERT2/Rosa-LSL-tdTomato mice revealed loss of EGFP expression (Supplemental Figure 4B). Taken together, this suggests that inhibiting Wnt signaling with C59 disturbs ISC self-renewal, but not ISC differentiation into TA cells. In agreement, a similar passive lineage commitment of ISCs into TA cells by in vivo targeting of both RSPO and Wnt receptors was recently reported (2).

We considered how Wnt inhibition might drive proliferation. Several lines of evidence suggested the involvement of the MAPK pathway. First, EGFR inhibition, which blocks 
A

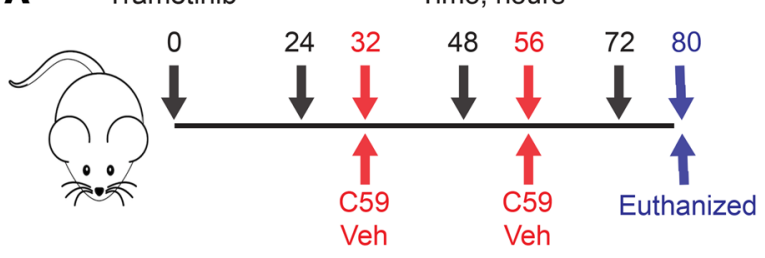

B
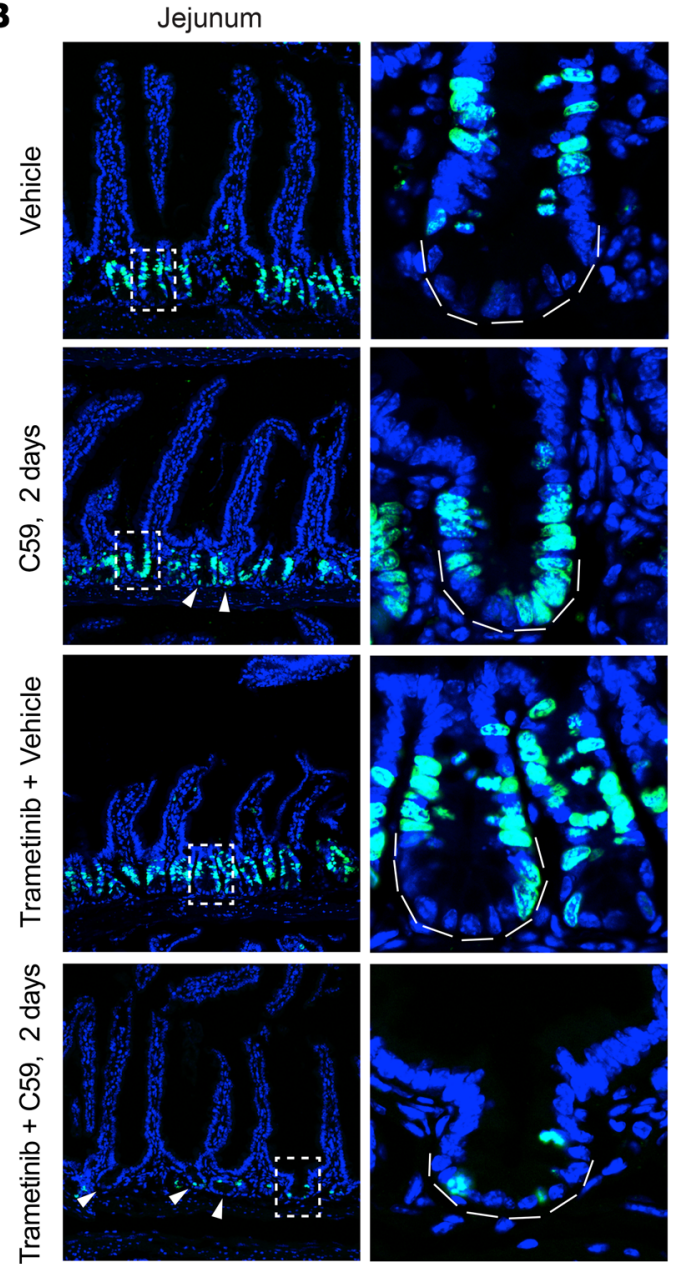

C
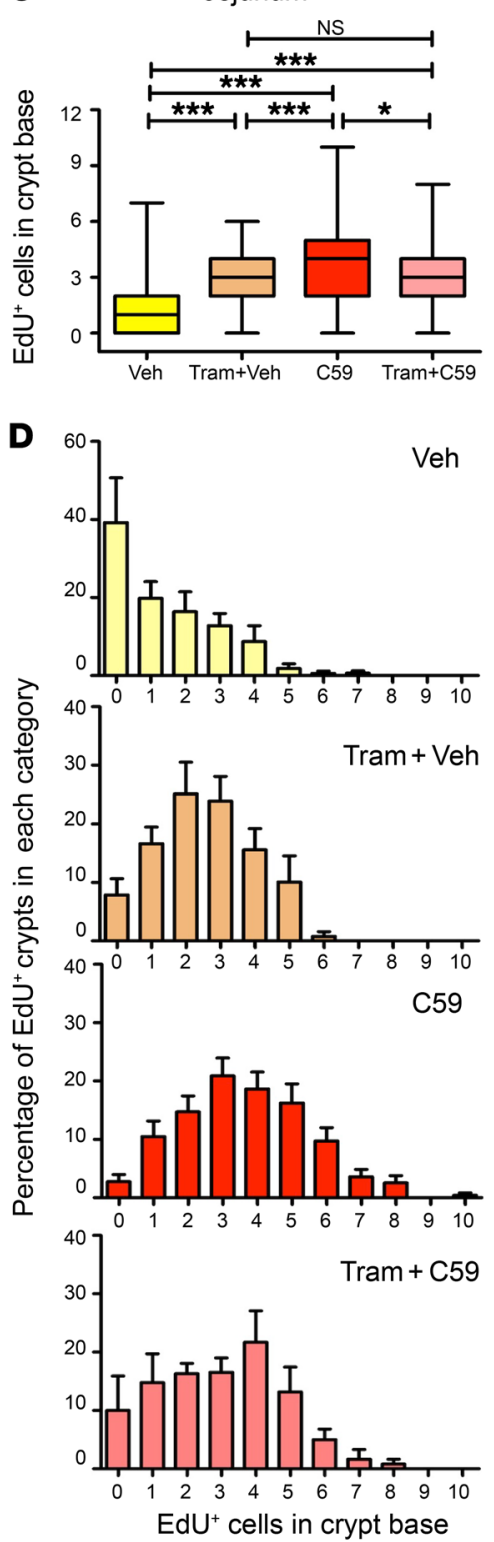

Figure 4. Suppressing MAPK signaling inhibits C59-induced proliferation of intestinal stem cells. (A) Schematic time line of trametinib, C59, and vehicle (Veh) administration in mice. Trametinib was administered (3 mg/ $\mathrm{kg}$, QD) 32 hours prior to administration of vehicle or $C 59$ (100 mg/kg, QD). A total of 4 doses of trametinib and 2 doses of $\mathrm{C} 59$ or vehicle were gavaged according to hourly time point (indicated by arrows) prior to euthanasia. (B) The combination of trametinib and C59 treatment blocked proliferation and impaired intestinal homeostasis. EdU was injected 2 hours prior to euthanasia into 4 groups of mice treated with either vehicle or C59 alone, or in combination with trametinib (Tram). Left panels show representative images of total EdU ${ }^{+}$ staining in jejunal crypts. Right panels show higher magnification of crypt as indicated. Scale bar, $25 \mu \mathrm{m}$. (C) Top graph shows the number of counts for $\mathrm{EdU}^{+}$cells within 10 cells in the base of the crypt. NS, nonsignificant; ${ }^{*} P$ $<0.05 ;{ }^{* *} P<0.001$, Kruskal-Wallis 1-way ANOVA test. (D) Trametinib blocks PORCN inhibition-induced burst of proliferation. The number of EdU ${ }^{+}$cells per 10 cells of the crypt base was determined. C59 treatment increased the number of crypts with 4 or more $\mathrm{EdU}^{+}$cells, and this was reversed by coadministration of trametinib. Vehicle, $n=7$. Trametinib plus vehicle, $n=6$. C59, $n=8$. Trametinib plus $C 59, n=6$. Error bar indicates SEM; 2 experimental replicates.
MEK-ERK signaling, was recently shown to suppress proliferation in intestinal organoid cultures (8). Second, high levels of p-ERK1/2, a measure of MAPK signaling, were reported in TA cells (21). Third, RSPO3, a Wnt sensitizer, was shown to inhibit MAPK signaling and block osteogenic differentiation of adipocyte stem cells in culture $(22,23)$. Therefore, the intestinal samples of C59- or vehicle-treated mice were stained for p-ERK1/2 by immunohistochemistry (IHC). The p-ERK1/2 staining was mainly positive in TA cells in vehicle-treated mice (Figure 3B). However, p-ERK1/2 expression was significantly increased in crypt base cells intercalated between Paneth cells in C59-treated mice (Figure 3, B and C and Supplemental Figure 4D). Thus, Wnt inhibition-induced ISC proliferation is associated with increased MAPK signaling.

To experimentally evaluate the role of MAPK signaling in intestinal proliferation following Wnt inhibition, mice were treated with trametinib (24), a potent MEK inhibitor, prior to
C59 treatment according to the time line shown in Figure 4A. In contrast to what was observed with C59 treatment alone, the combination of trametinib and C59 led to a rapid decline in the health of the treated mice, as evidenced by decreased activity, rapid weight loss, and ascites. IHC with p-ERK1/2 antibody confirmed that trametinib reduced MAPK signaling (Supplemental Figure 5C). The combination of trametinib and C59 was not toxic for ISCs as there was no staining for the apoptotic marker CAS3 in crypt bases (Supplemental Figure 5D). In agreement, total proliferation as judged by counts of EdU' cells was significantly reduced in the trametinib plus C59 group as compared with the vehicle or C59 groups (Supplemental Figure 5A). Interestingly, trametinib alone increased baseline EdU uptake at the base of the crypt, which we suspect was secondary to enteropathy in the villi (25) (Figure 4, A-D). Nevertheless, ISC proliferation in mice from the trametinib plus C59 group was similar to trametinib treatment alone and significantly low- 
er than C59-treated mice. Taken together, these findings suggest that MEK inhibition suppresses the proliferation caused by acute Wnt inhibition (Figure 4, C and D). This effect was seen throughout the majority of the small intestine with the exception of small parts of the proximal and distal small intestine (Supplemental Figure 1B and Supplemental Figure 5B). Admittedly, other signaling mechanisms might coexist with ERK1/2 activation, and/or compensate for ERK inhibition, for example, activation of ERK5 (25). With this caveat acknowledged, inhibition of MEK prior to Wnt inhibition blocked proliferation and differentiation of ISCs into TA cells and impaired intestinal homeostasis shortly after C59 administration. This model is consistent with studies showing that increased MAPK signaling in the intestine due to BRAFV600 mutation promotes loss of ISCs and increases differentiation $(26,27)$.

Taken together, our data not only demonstrate an unanticipated role of Wnt suppressing ISC proliferation, but also supports an interaction between Wnt and MAPK signaling in vivo. We propose that Wnt signaling maintains quiescent ISC pools through suppression of the MAPK pathway in the intestine. These concepts will be useful in designing combination therapies for cancer and in regenerative medicine.

\section{Methods}

Mice strains and drug administration. Lgr5-EGFP-IRES-CreER ${ }^{T 2}$ (stock 008875), Bmi1-creER ${ }^{T}$ (stock 010531), C57BL/6J (stock 000664) and Rosa-LSL-tdTomato (stock 007905) were purchased from the Jackson laboratory. Lgr5-EGFP_IRES-creER $R^{T 2}$ and Bmi1-creER $R^{T}$ were crossed into Rosa-LSL-tdTomato to generate Lgr5-EGFP-IRES - creER $^{T 2}(+,-) /$ RosaLSL-tdTomato(+,-) or Bmi1-CreER ${ }^{T}(+,-) /$ Rosa-LSL-tdTomato(+,-) mice for lineage tracing experiments. Tamoxifen (Sigma, catalog T5648-1G) was dissolved in corn oil (catalog C8267, $20 \mathrm{mg} / \mathrm{ml}$ ) through vigorous shaking at $37^{\circ} \mathrm{C}$. Final doses of $50 \mathrm{mg} / \mathrm{kg}$ and $100 \mathrm{mg} / \mathrm{kg}$ were injected intraperitoneally into Bmi1-creER ${ }^{T}(+,-) /$ Rosa-LSL-tdTomato(+,-) and Lgr5-EGFP-IRES - creER ${ }^{T 2}(+,-) /$ Rosa-LSL-tdTomato(+,-) mice respectively. PORCN inhibitor C59 was synthesized by the Duke University Small Molecule Synthesis facility and administered as described (28). Trametinib was purchased from Chemitek (catalog CT-GSK212) and dissolved in DMSO $(10 \mathrm{mg} / \mathrm{ml})$. The trametinib stock was adjusted to a final concentration of $0.3 \mathrm{mg} / \mathrm{ml}$ using vehicle solution ( $0.5 \%$ methylcellulose plus $0.2 \%$ Tween-80) and administered at $3 \mathrm{mg} / \mathrm{kg}$ dose by oral gavage.

Cell proliferation assay. Cell proliferation in vivo was measured by administering EdU 2 hours prior to euthanasia and measuring incor- poration using EdU and ClickiT EdU Alexa Fluor 488 and 647 Imaging Kits from Life Technologies (catalog E10187, C10337, and C10640 respectively) as previously described (6). All samples were analyzed using a Leica SP5 inverted confocal microscope.

Images for different parts of the intestine were obtained based on the aforementioned criteria for tissue preparation and antibody staining. Quantification of EdU' cells was done blindly on 10 cells in the bottom of the crypt based on cell position 0, as shown by Supplemental Figure 1C. We defined TA cells based on position within the crypt, EdU incorporation, and expression of cell cycle markers.

Statistics. Data were analyzed using Prism 6 software (GraphPad Software).

Study approval. The Duke University IACUC (Durham, NC) approved all animal studies.

\section{Author contributions}

ZK and GG were responsible for the study concept and design, acquisition, analysis, and interpretation of data, and drafting the manuscript. HZ was responsible for mouse colony management and acquisition and analysis of data. AH was responsible for analysis and scoring of IHC samples. CMC and DMV were responsible for the study concept and design, study supervision, obtaining funding, and drafting the manuscript.

\section{Acknowledgments}

We thank Yasheng Gao for technical assistance. We are grateful for technical support from the Duke University light microscopy core facility and the Duke University research histopathology laboratory. This work was supported by grant R01CA123031 (to $\mathrm{CMC}$ ). This research is supported in part by the National Research Foundation Singapore and administered by the Singapore Ministry of Health's National Medical Research Council under the STAR Award Program (to DMV).

Address correspondence to: Zahra Kabiri or Christopher M. Counter, Department of Pharmacology and Cancer Biology, Duke University Medical Center, 308 Research Drive, LSRC C229 (Z. Kabiri) or LSRC C225 (C.M. Counter), Durham, North Carolina 27710, USA. Phone: 919.684.1967; Email: zahrakabiri.md@gmail.com (Z. Kabiri). Phone: 919.684.9890; Email: chris.counter@duke.edu (C.M. Counter). Or to: David M. Virshup, Program in Cancer and Stem Cell Biology, Duke-NUS Medical School, 8 College Road, Singapore 169857. Phone: 65.6516.6954; Email: david.virshup@ duke-nus.edu.sg.
1. Kuhnert F, et al. Essential requirement for Wnt signaling in proliferation of adult small intestine and colon revealed by adenoviral expression of Dickkopf-1. Proc Natl Acad Sci U S A. 2004;101(1):266-271.

2. Yan KS, et al. Non-equivalence of Wnt and R-spondin ligands during Lgr5+ intestinal stem-cell selfrenewal. Nature. 2017;545(7653):238-242.

3. Storm EE, et al. Targeting PTPRK-RSPO3 colon tumours promotes differentiation and loss of stemcell function. Nature. 2016;529(7584):97-100.

4. van Es JH, et al. A critical role for the Wnt effector Tcf4 in adult intestinal homeostatic self-renewal.
Mol Cell Biol. 2012;32(10):1918-1927.

5. Fevr T, Robine S, Louvard D, Huelsken J. Wnt/ beta-catenin is essential for intestinal homeostasis and maintenance of intestinal stem cells. $\mathrm{Mol}$ Cell Biol. 2007;27(21):7551-7559.

6. Kabiri Z, et al. Stroma provides an intestinal stem cell niche in the absence of epithelial Wnts. Development. 2014;141(11):2206-2215.

7. Greicius G, et al. PDGFR $\alpha+$ pericryptal stromal cells are the critical source of Wnts and RSPO3 for murine intestinal stem cells in vivo. Proc Natl Acad Sci U S A. 2018;115(14):E3173-E3181.

8. Basak O, Beumer J, Wiebrands K, Seno H, van
Oudenaarden A, Clevers H. Induced quiescence of Lgr5+ stem cells in intestinal organoids enables differentiation of hormone-producing enteroendocrine cells. Cell Stem Cell. 2017;20(2):177-190.e4.

9. Jin Y, et al. EGFR/Ras signaling controls drosophila intestinal stem cell proliferation via capicua-regulated genes. PLoS Genet. 2015;11(12):e1005634.

10. Biteau B, Jasper H. EGF signaling regulates the proliferation of intestinal stem cells in Drosophila. Development. 2011;138(6):1045-1055.

11. Miyoshi H, et al. Prostaglandin E2 promotes intestinal repair through an adaptive cel- 
lular response of the epithelium. $E M B O J$. 2017;36(1):5-24.

12. Proffitt KD, et al. Pharmacological inhibition of the Wnt acyltransferase PORCN prevents growth of WNT-driven mammary cancer. Cancer Res. 2013;73(2):502-507.

13. Snippert HJ, et al. Intestinal crypt homeostasis results from neutral competition between symmetrically dividing Lgr5 stem cells. Cell. 2010;143(1):134-144.

14. Barker N, et al. Identification of stem cells in small intestine and colon by marker gene Lgr5. Nature. 2007;449(7165):1003-1007.

15. Madan B, et al. Wnt addiction of genetically defined cancers reversed by PORCN inhibition. Oncogene. 2016;35(17):2197-2207.

16. Liu J, et al. Targeting Wnt-driven cancer through the inhibition of Porcupine by LGK974. Proc Natl Acad Sci U S A. 2013;110(50):20224-20229.

17. van der Flier LG, Haegebarth A, Stange DE, van de Wetering $\mathrm{M}$, Clevers H. OLFM4 is a robust marker for stem cells in human intestine and marks a subset of colorectal cancer cells. Gastroenterology. 2009;137(1):15-17.

18. Schuijers J, et al. Ascl2 acts as an R-spondin/Wntresponsive switch to control stemness in intestinal crypts. Cell Stem Cell. 2015;16(2):158-170.

19. Sangiorgi E, Capecchi MR. Bmi1 is expressed in vivo in intestinal stem cells. Nat Genet. 2008;40(7):915-920.

20. Zhu Y, Huang YF, Kek C, Bulavin DV. Apoptosis differently affects lineage tracing of Lgr5 and Bmi1 intestinal stem cell populations. Cell Stem Cell. 2013;12(3):298-303.

21. Heuberger J, Kosel F, Qi J, Grossmann KS, Rajewsky K, Birchmeier W. Shp2/MAPK signaling controls goblet/paneth cell fate decisions in the intestine. Proc Natl Acad Sci U S A. 2014;111(9):3472-3477.

22. Zhang M, et al. RSPO3-LGR4 regulates osteogenic differentiation of human adipose-derived stem cells via ERK/FGF signalling. Sci Rep. 2017;7:42841.

23. Sundaram GM, et al. EGF hijacks miR-198/
FSTL1 wound-healing switch and steers a twopronged pathway toward metastasis. J Exp Med. 2017;214(10):2889-2900.

24. Gilmartin AG, et al. GSK1120212 (JTP-74057) is an inhibitor of MEK activity and activation with favorable pharmacokinetic properties for sustained in vivo pathway inhibition. Clin Cancer Res. 2011;17(5):989-1000.

25. de Jong PR, et al. ERK5 signalling rescues intestinal epithelial turnover and tumour cell proliferation upon ERK1/2 abrogation. Nat Commun. 2016;7:11551.

26. Tong K, et al. Degree of tissue differentiation dictates susceptibility to BRAF-driven colorectal cancer. Cell Rep. 2017;21(13):3833-3845.

27. Riemer P, et al. Transgenic expression of oncogenic BRAF induces loss of stem cells in the mouse intestine, which is antagonized by $\beta$-catenin activity. Oncogene. 2015;34(24):3164-3175.

28. Kabiri Z, et al. Wnts are dispensable for differentiation and self-renewal of adult murine hematopoietic stem cells. Blood. 2015;126(9):1086-1094. 